\title{
STUDY OF SILICON THICKNESS OPTIMIZATION FOR LSST ${ }^{*}$
}

\author{
P. O'Connor ${ }^{\mathrm{a}}$, V. Radeka ${ }^{\mathrm{a}}$, D. Figer ${ }^{\mathrm{b}}$, J. G. Geary ${ }^{\mathrm{c}}$, D. K. Gilmore ${ }^{\mathrm{d}}$, J. Oliver ${ }^{\mathrm{e}}$, \\ C. W. Stubbs ${ }^{f}$, P. Z. Takacs ${ }^{\mathrm{a}}$, and J. A. Tyson ${ }^{\mathrm{g}}$ \\ ${ }^{a}$ Brookhaven National Laboratory, Upton, NY 11973 \\ ${ }^{\mathrm{b}}$ Space Telescope Science Institute, Baltimore, MD 21218 \\ ${ }^{c}$ Harvard-Smithsonian Center for Astrophysics, Cambridge, MA 02138 \\ ${ }^{\mathrm{d} S t a n f o r d ~ L i n e a r ~ A c c e l e r a t o r ~ C e n t e r, ~ S t a n f o r d, ~ C A ~}$ \\ ${ }^{\mathrm{E} H a r v a r d}$ Univ. Lab. for Particle Physics and Cosmology, Cambridge, MA 02138 \\ ${ }^{f}$ Department of Physics, Harvard Univ., Cambridge, MA 02138 \\ ${ }^{\mathrm{g}}$ University of California, Davis, CA 95616
}

May, 2006

*This manuscript has been authored by Brookhaven Science Associates, LLC under Contract No. DE-AC02-98CH10886 with the U.S. Department of Energy. The United States Government retains, and the publisher, by accepting the article for publication, acknowledges, a world-wide license to publish or reproduce the published form of this manuscript, or allow others to do so, for the United States Government purposes. 


\section{DISCLAIMER}

This work was prepared as an account of work sponsored by an agency of the United States Government. Neither the United States Government nor any agency thereof, nor any of their employees, nor any of their contractors, subcontractors, or their employees, makes any warranty, express or implied, or assumes any legal liability or responsibility for the accuracy, completeness, or any third party's use or the results of such use of any information, apparatus, product, or process disclosed, or represents that its use would not infringe privately owned rights. Reference herein to any specific commercial product, process, or service by trade name, trademark, manufacturer, or otherwise, does not necessarily constitute or imply its endorsement, recommendation, or favoring by the United States Government or any agency thereof or its contractors or subcontractors. The views and opinions of authors expressed herein do not necessarily state or reflect those of the United States Government or any agency thereof. 


\title{
Study of Silicon Thickness Optimization for LSST
}

\section{P. O'Connor ${ }^{\mathrm{a}^{*}}$, V. Radeka ${ }^{\mathrm{a}}$, D. Figer ${ }^{\mathrm{b}}$, J.G. Geary, D.K. Gilmore ${ }^{\mathrm{d}}$, J. Oliver ${ }^{\mathrm{e}}$, C.W. Stubbs ${ }^{\mathrm{f}}$, P.Z.} Takacs ${ }^{\mathrm{a}}$, J.A. Tyson ${ }^{\mathrm{g}}$

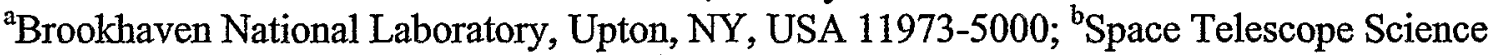
Institute, 3700 San Martin Drive, Baltimore, MD 21218; ${ }^{\circ}$ Harvard-Smithsonian Center for Astrophysics, 60 Garden St., Cambridge MA 02138; 'Stanford Linear Accelerator Center, Stanford, CA; ${ }^{\mathrm{e} H a r v a r d}$ University, Laboratory for Particle Physics and Cosmology, 18 Hammond St., Cambridge, MA 02138; 'Department of Physics, Harvard University, 17 Oxford St., Cambridge,

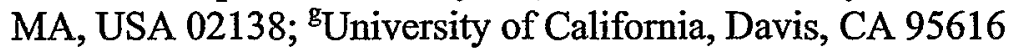

\begin{abstract}
Sensors for the LSST camera require high quantum efficiency (QE) extending into the near-infrared. A relatively large thickness of silicon is needed to achieve this extended red response. However, thick sensors degrade the point spread function (PSF) due to diffusion and to the divergence of the fast $f / 1.25$ beam. In this study we examine the tradeoff of $\mathrm{QE}$ and PSF as a function of thickness, wavelength, temperature, and applied electric field for fully-depleted sensors. In addition we show that for weakly absorbed long-wavelength light, optimum focus is achieved when the beam waist is positioned slightly inside the silicon.
\end{abstract}

Keywords: LSST, quantum efficiency, point spread function, overdepletion

\section{INTRODUCTION}

The LSST focal plane is the largest ever proposed for an astronomical instrument. The demands of the science to be done and the nature of the cadence and very wide field preclude the use of any existing imager, so a custom device must be developed. Both CCD and PIN-CMOS imagers are being considered. Of central importance are high quantum efficiency (QE) extending into the near infrared and small point spread function (PSF). To achieve high QE in the nearIR, the sensor must be thick because the absorption depth of silicon increases rapidly in this wavelength range. However, increasing detector thickness degrades the spatial resolution of the sensor due to two effects. Diffusion of the photogenerated charge increases because of the longer transit time to the collecting electrode. Thick sensors also require sufficient substrate bias to fully deplete the device; otherwise, lateral diffusion in the undepleted field-free region severely degrades the PSF. A second cause of PSF broadening results from the fast focal ratio of the LSST optics (f/1.2). For red wavelengths where the absorption length is comparable to or greater than the sensor thickness, the light becomes defocused before it is fully absorbed, further broadening the PSF.

This study is organized as follows:

- Section 2: quantum efficiency as a function of wavelength, temperature, and thickness

- Section 3: point spread function due to diffusion and beam divergence

- Section 4: optimization of thickness using point source limiting magnitude as a figure of merit

This manuscript has been co-authored by employees of Brookhaven Science Associates, LLC under Contract No. DE-AC0298CH10886 with the U.S. Department of Energy. The LSST design and development activity is supported by the National Science Foundation under Scientific Program Order No. 9 (AST-0551161) through Cooperative Agreement AST-0132798. Portions of this work were performed in part under Department of Energy contracts DE-AC02-76SF00515, DE-FG02-91ER40677 and W7405-Eng-48. Additional funding comes from private donations, in-kind support at Department of Energy laboratories and other LSSTC Institutional Members.

* poc@bnl.gov; phone 1631 344-7577; fax 1631 344-5773; http://www.inst.bnl.gov/programs/microelec/ 


\section{QUANTUM EFFICIENCY}

We distinguish here between internal $\mathrm{QE}$, the device efficiency after light enters the detector, and total $\mathrm{QE}$, which includes reflection losses due to dielectric mismatch. Our focus in this paper is on internal $\mathrm{QE}$.

\subsection{Absorption length in silicon}

The absorption length is defined as the depth at which the light intensity falls to $1 / \mathrm{e}$ of the original incident intensity. We adopt the empirical model of Rajkanan [1] to find the absorption length of silicon as a function of wavelength and temperature [Fig. 1]. Note that absorption length increases rapidly for photon energies near the band gap of silicon (about $1000 \mathrm{~nm}$ ). There is also a strong increase of absorption with temperature, especially in the red, since transitions near the indirect band gap must be phonon-assisted to conserve momentum.

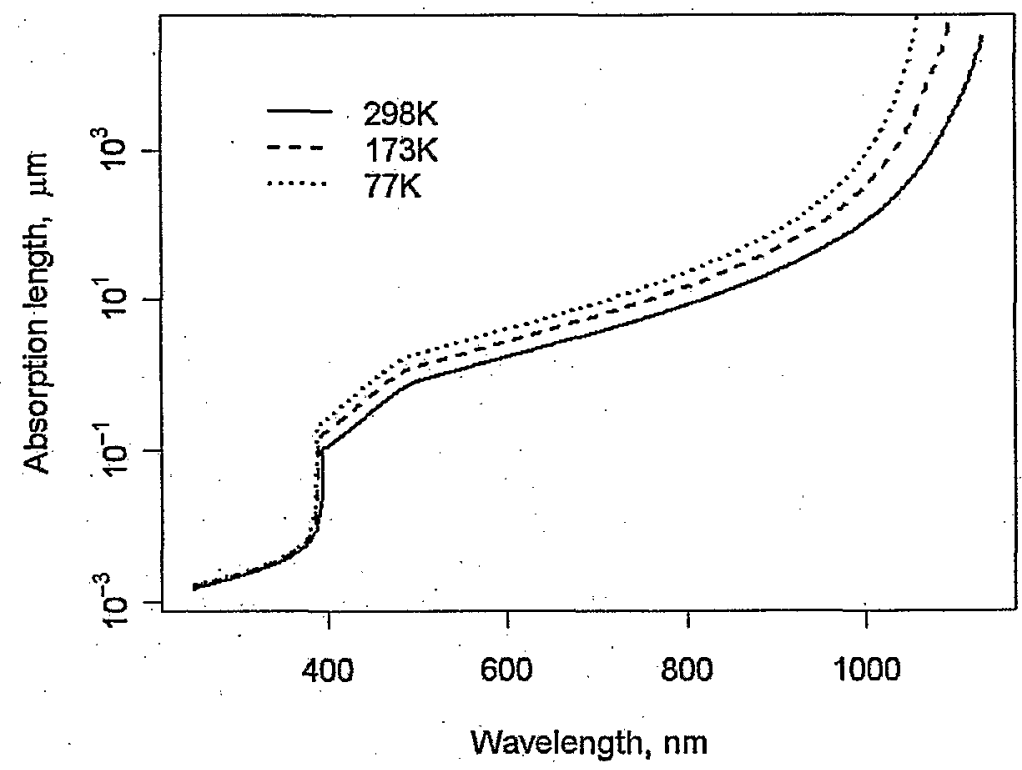

Figure 1. Absorption length in silicon, after Reference [1]. Long-wavelength absorption length is strongly temperaturedependent.

\subsection{Quantum efficiency}

The LSST specifications for total quantum efficiency are as follows [2]:

Table 1. LSST quantum efficiency requirements

\begin{tabular}{lll}
\hline Wavelength & \multicolumn{2}{l}{ Quantum Efficiency } \\
\hline & Allowable & Target \\
$400 \mathrm{~nm}$ & $55 \%$ & $60 \%$ \\
600 & 80 & 85 \\
800 & 80 & 85 \\
900 & 60 & 85 \\
1000 & 25 & 45 \\
\hline
\end{tabular}


Sensor quantum inefficiency arises from reflection loss, incomplete charge collection (usually a negligible effect), and incomplete light absorption. In this study we only model the effect of incomplete absorption. The reflectivity properties of the final LSST sensors will depend on the antireflection coating used on the illuminated surface and on the interface properties on the opposite (charge-collecting) side, which are not known at this point. Charge collection is expected to be near unity over most of the wavelength range, but surface defects will cause a falloff toward the blue end where absorption takes place very near the surface. For the red and near-IR region, reflection losses will reduce the $Q E$ and multiple internal reflections will cause interference fringes in the optical response (although for thick sensors and low $\mathrm{f}$ number the fringing is reduced). Hence the internal quantum efficiency reported here should be considered an upper limit.

Figure 2 shows the modeled quantum efficiency at longer wavelengths for different sensor thicknesses at a temperature of $-100^{\circ} \mathrm{C}$.

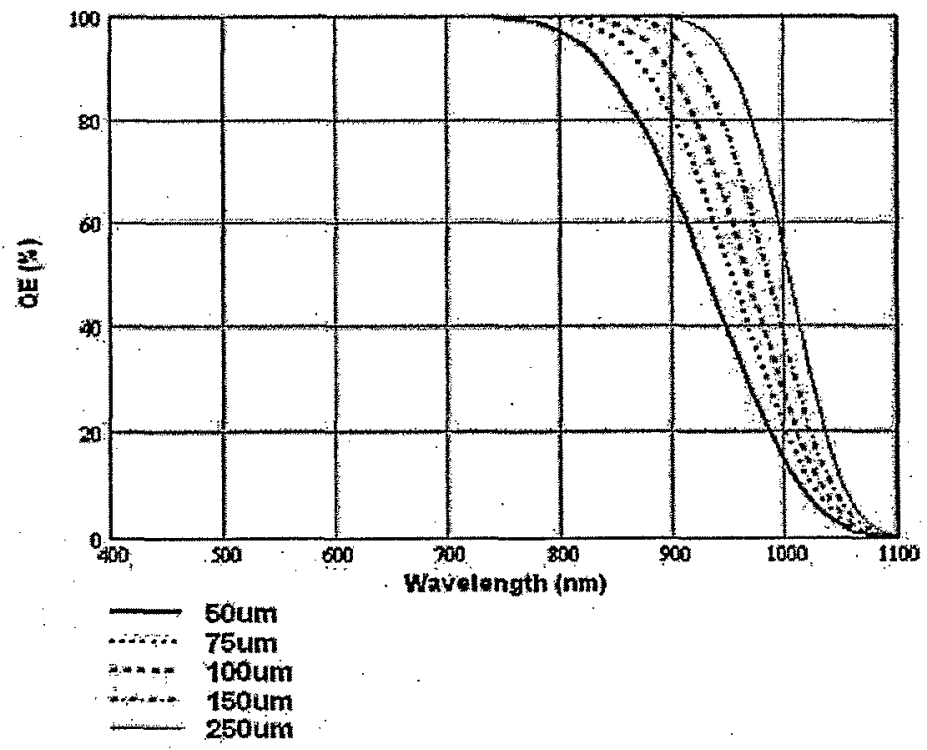

Figure 2. Internal quantum efficiency at $173 \mathrm{~K}$ as a function of wavelength for several sensor thicknesses. Reflection and charge collection losses not modeled.

For the critical 1000-nm wavelength, the dependence of internal $\mathrm{QE}$ on temperature and sensor thickness is shown by the contour plot in Figure 3 . If the focal plane is operated at a temperature of $-100 \mathrm{C}$ (choice of temperature is dependent on dark current characteristics), a silicon thickness of no less than $100 \mu \mathrm{m}$ will be needed to meet the LSST minimum acceptable $\mathrm{QE}$ at $1000 \mathrm{~nm}$. Note that at $\lambda=1000 \mathrm{~nm}$ the relative $\mathrm{QE}$ varies by about $1 \%$ per degree $\mathrm{C}$. 


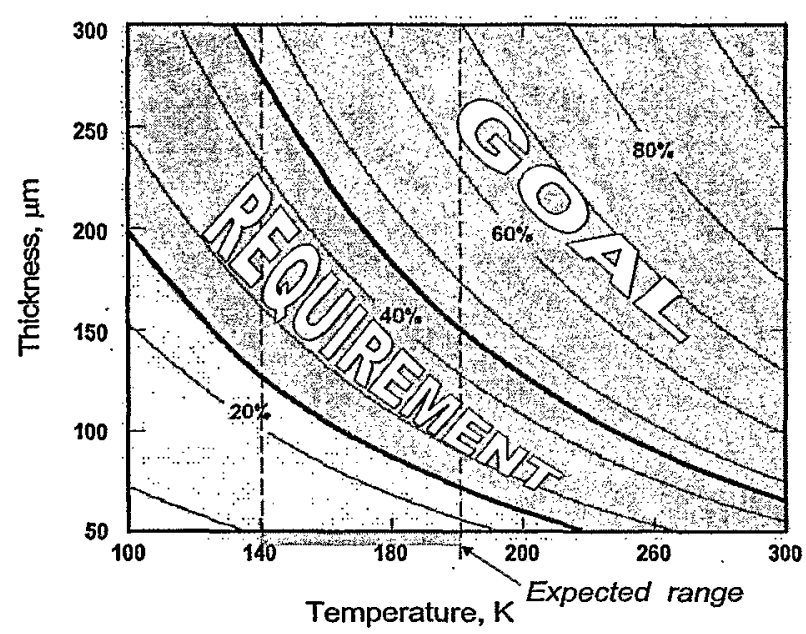

Figure 3. Internal $\mathrm{QE}$ at $\lambda=1000 \mathrm{~nm}$ (solid contours) as a function of sensor thickness and temperature.

\section{POINT SPREAD FUNCTION}

Several of the science missions of the LSST depend on its ability to resolve faint point sources. The signal-to-noise ratio for a point source depends inversely on the PSF, since the smaller the area over which the image is spread, the less sky noise is acquired with the signal. Contributions to the PSF come from aberrations of the telescope optics (including fabrication and alignment tolerances), atmospheric seeing at the site, and detector contributions. The LSST specification for the detector contribution to the point spread function is [2]:

\begin{tabular}{lll}
\hline & Allowable & Target \\
\hline Pixel FWHM (charge spreading) & $<10 \mu \mathrm{m}$ & $<7.5 \mu \mathrm{m}$ \\
\hline
\end{tabular}

(In subsequent discussion of we will assume an approximately Gaussian PSF, for which the allowable and target rms values are 4.25 and $3.19 \mu \mathrm{m}$ respectively.)

The LSST plate scale is $50.9 \mu \mathrm{m}$ per arcsecond, and the pixel size is $10 \mu \mathrm{m}$.

At short wavelengths, the main contribution to charge spreading comes from diffusion. At near-IR wavelengths, there is additional broadening due to the divergent "cone" of light that enters the sensor. The light becomes progressively defocused and the deeper the point of absorption, the wider the effective spot size. These effects will be analyzed in the next two sections.

\subsection{Charge Diffusion}

Visible photons are absorbed in a thin layer near the illuminated (back) side of the sensor and the resulting charges move to the electrode side under the influence of the applied electric field. To achieve small point spread function it is important to apply a bias that will deplete the entire sensor thickness. If not, there will be a field-free, neutral layer of thickness $d_{F F}$ near the illuminated face of the sensor. Charges generated in the field-free region diffuse isotropically and will have a profile of rms width $\approx d_{F F}$ when they encounter the electric field that sweeps them to the collecting side of the sensor. The bias needed to fully deplete a device of thickness $d$ is

$$
V_{d e p l}=\frac{q d^{2} N}{2 \varepsilon_{S i}},
$$


where $N$ is the density of donor or acceptor dopants in the substrate, $\varepsilon_{S i}$ is the permittivity of silicon, and $q$ is the electron charge. For high-resistivity silicon with a dopant density in the range of $10^{12} \mathrm{~cm}^{-3}$, about $10 \mathrm{~V}$ is required to deplete a thickness of $100 \mu \mathrm{m}$. In terms of material resistivity, for a $5 \mathrm{k} \Omega$-cm substrate $\sim 7 \mathrm{~V}$ is required in $\mathrm{n}$-type and $\sim 20 \mathrm{~V}$ in p-type for full depletion.

Using a one-dimensional approximation, the electric field in an overdepleted sensor of thickness $d$ varies linearly with distance $z$ from the illuminated surface as

$$
E(z)=E_{\min }+2 V_{d e p t} z / d^{2}
$$

where $E_{\min }=\left(V_{o p}-V_{d e p}\right) / d$ is the field at the illuminated surface, $V_{o p}$ is the operating voltage, and $V_{d e p t}$ is the depletion voltage given by Eq. (2). The rms diffusion for carriers traversing the full sensor thickness is

$$
\sigma_{D}=\sqrt{2 D t_{d r}},
$$

where $t_{d r}=\int_{0}^{d} d z / v$ is the drift time of carriers moving with velocity $v$ in the electric field.

In an overdepleted sensor and high resistivity material $(\rho>10000 \Omega-\mathrm{cm})$ the velocity is nearly constant. Assuming initially a constant mobility, the drift velocity is given by $\nu=\mu E_{a v}=\mu V_{a p p l} / d$, where $\mu$ is the mobility of minority carriers and $V_{o p}$ is the applied voltage across the sensor. Then using the Einstein relation $D=k T \mu / q$ and (3) we find a simplified expression for the diffusion

$$
\sigma_{D} \approx \sqrt{\frac{2 k T}{q E}}=d \sqrt{\frac{2 k T}{q V_{o p}}}
$$

Equation (4) does not depend on any silicon properties and is useful for first-order estimates of diffusion, but only in over depletion, $V_{\text {op }} / V_{\text {depp }}>2$.

In the general case the diffusion calculation has to take into account the non-uniform electric field (2), as well as fielddependent carrier velocity and field-dependent longitudinal and transverse diffusion coefficients and their temperature dependence. In the special case of interest to LSST, the sensors will have to be operated well overdepleted and then the effects of the slightly non-uniform electric field become negligible. However, the carrier velocity saturation effects are important and have to be taken into account. There is an increase in diffusion due to longer carrier drift time than expected in the constant mobility case, while there is a compensating decrease in the transverse diffusion coefficient due to the streamlining effect of the electric field on the carriers in random + drift motion. These effects have been included in all the calculations of the expected diffusion contribution to the PSF discussed in the remainder of this study. The velocity effects on diffusion will be discussed in a separate note. For a temperature of $173 \mathrm{~K}$ and $5 \mathrm{kV} / \mathrm{cm}$ the two opposing effects result in an overall diffusion increase of a factor of about 1.15 for electrons (1.3 for holes) compared to the simple approximation (4).

Long-wavelength light generates carrièrs at varying depths in the silicon, so their diffusion has to be weighted by the absorption probability:

$$
\sigma_{D}(\lambda)=\frac{\int_{0}^{d} e^{-z / I_{a b s}(\lambda)} \sigma_{D}(z) d z}{\int_{0}^{d} e^{-z / l_{a b s}(\lambda)} d z},
$$

where $\sigma_{D}(z)$ is the diffusion experienced by a carrier generated at a distance $z$ from the entrance window of a sensor whose total thickness is $d$.

Figure 4 shows the PSF contours for high-resistivity p-type silicon on the thickness-voltage plane, with the effects of high and nonuniform electric field taken into account. Note that the voltage required to meet the LSST PSF target increases as the square of the silicon thickness, and is about four times greater than the full-depletion voltage for high- 
resistivity material. For silicon with resistivity in this range, the rms diffusion exhibits a temperature coefficient of $0.007 \mu \mathrm{m} /{ }^{\circ} \mathrm{C}$.

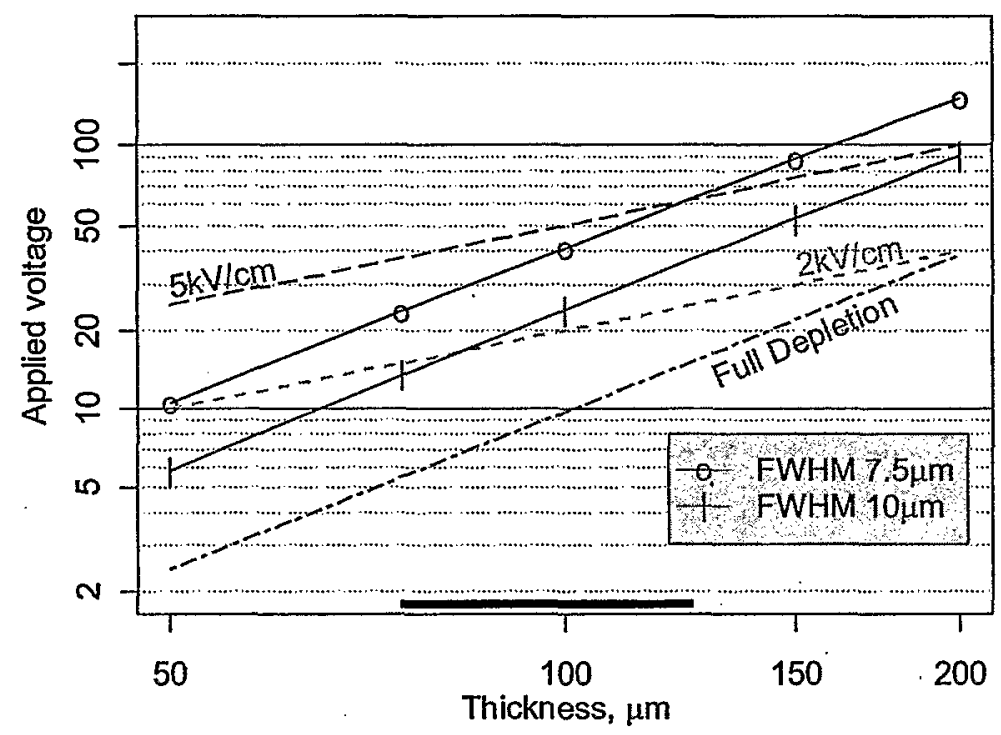

Figure 4. Diffusion contours for short-wavelength light in p-type silicon, $10 \mathrm{k} \Omega-\mathrm{cm}$, at $173 \mathrm{~K}$. Dashed lines show average electric fields and the full depletion voltage. Carrier velocity saturation effects have been included in the diffusion calculation. The LSST acceptable is PSF $=10 \mu \mathrm{m}$ FWHM $(4.25 \mu \mathrm{m} \mathrm{ms})$, the target is PSF $=7.5 \mu \mathrm{m}$ $(3.2 \mu \mathrm{m} \mathrm{rms})$.

\subsection{PSF broadening due to divergent optical beam in silicon}

In a low-f number optical system like LSST, light is incident on the sensor at large angles from the normal. Figure 5 shows an optical raytrace result from the LSST $\mathrm{f} / 1.2$ configuration. Because of the high index of refraction of silicon, the light "cone" has a smaller half-angle inside the silicon than in air. The lower half of the figure shows the absorption of light of two wavelengths. For long wavelengths where the light absorption length $l_{a b s}$ is much greater than the sensor thickness, the light is absorbed almost uniformly, while shorter wavelength light is strongly absorbed at the surface. 


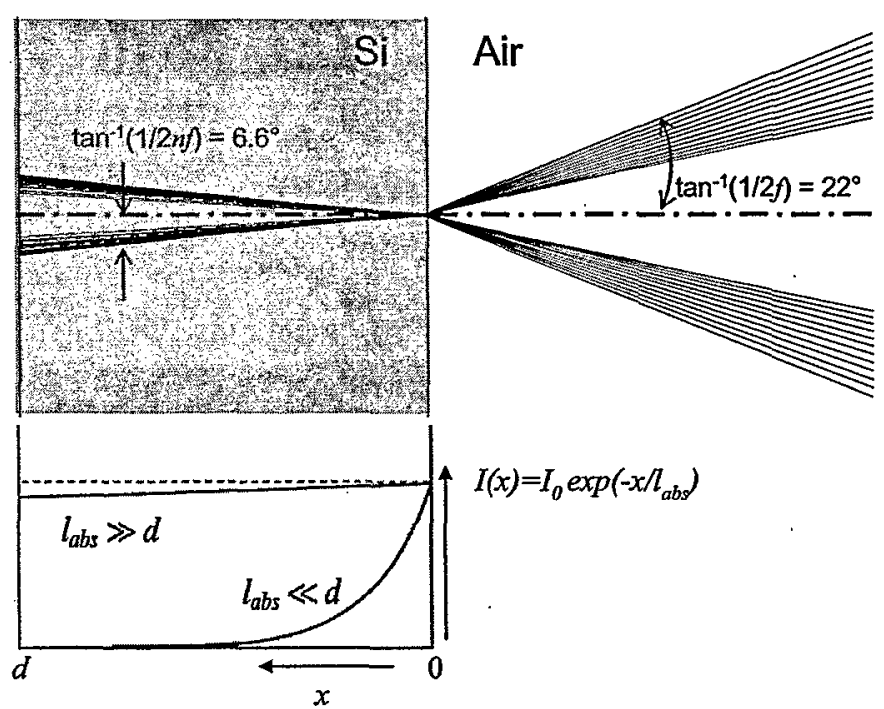

Figure 5. Optical raytrace showing refraction and absorption of light entering the silicon sensor. Light is incident from the right and focus is at the sensor illuminated surface.

Two methods were used to study the effect of beam divergence on PSF.

\subsubsection{Raytrace method}

Monte-Catlo simulations were performed for absorption lengths of $2,10,50,100,200$, and $300 \mu \mathrm{m}$ and for sensor thicknesses from 50 to $250 \mu \mathrm{m}$. For each configuration, ten thousand rays were traced into a thick silicon layer and the depth of conversion was selected according to the appropriate exponential probability distribution. The resulting ionization distribution was then projected onto the collecting surface, with the effect of diffusion optionally included based on the drift distance of each electron and using the expressions developed in subsection 3.1 .

This method can also be used to study the optimum position of the focal plane. Because of the weak absorption at long wavelengths, the projected charge distribution on the electrode side of the sensor can be made smaller by displacing the focal plane in the direction of the incoming light. Figure 6 illustrates the effect. When the sensor is displaced towards the incoming rays, long-wavelength light comes to a focus within the body of the sensor and the projection of the generated charge forms a smaller spot. The amount of focal plane displacement is highly sensitive to wavelength and also depends on temperature. 

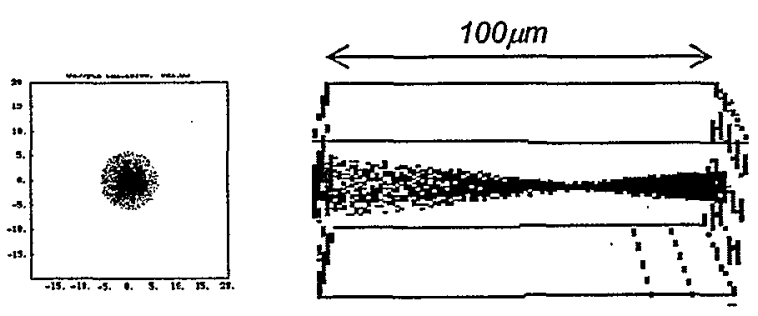

i.

FP displacement:

+10 um
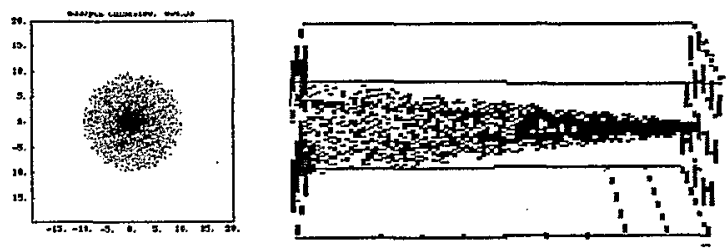

$0 \mu \mathrm{m}$
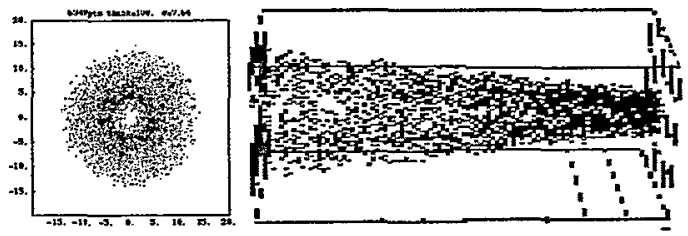

$-10 \mu \mathrm{m}$

Figure 6. Absorption of long-wavelength light in a $100 \mu \mathrm{m}$-thick sensor. Focal plane position shown displaced by 0 , and \pm 10 microns from best short-wavelength focus. Spot diagrams (with no diffusion) shown on the left.

\subsubsection{Geometrical method}

An analytic estimate of the PSF broadening from beam divergence can also be made, by weighting the radius of the light cone by the absorption probability at a depth $z$ in the sensor. The telescope images a point-source to a spot modeled by a Gaussian profile of width $\sigma_{\text {tel }}$. The silicon sensor is initially introduced with its entrance face at $z=0$ and refracts the light as shown in Fig. 5; positive and negative displacements of the sensor produce light distributions as shown in Fig. 6. We model the geometric width of the light spot as a function of the displacement $\delta$ and the distance from the entrance face $z$ as:

$$
\begin{aligned}
\sigma(\delta, z) & =\sigma_{\text {tel }}+\frac{|z-n \delta|}{2 n f}, \quad \delta>0 \\
& =\sigma_{\text {tel }}+\frac{|\delta|}{2 f}+\frac{z}{2 n f}, \quad \delta<0
\end{aligned}
$$

where $n$ is the refractive index of silicon and $f$ is the focal ratio of the telescope. Equation (6) accounts for the increased width of the beam at the sensor surface and the appropriate converging and diverging light "cones" in the sensor. Following Groom $[3,4]$ we form the weighted average of the beam width over the exponential absorption through the sensor:

$$
\sigma_{d i v}^{2}(\delta)=\frac{\int_{0}^{d} \sigma^{2}(\delta, z) e^{\frac{-z}{l_{b b s}}} d z}{\int_{0}^{d} e^{\frac{-z}{I_{a b s}}} d z}
$$


The geometry is illustrated in Figure 7. This method was found to give very good agreement with the raytrace results over a range of thickness, absorption length, and focal displacement.

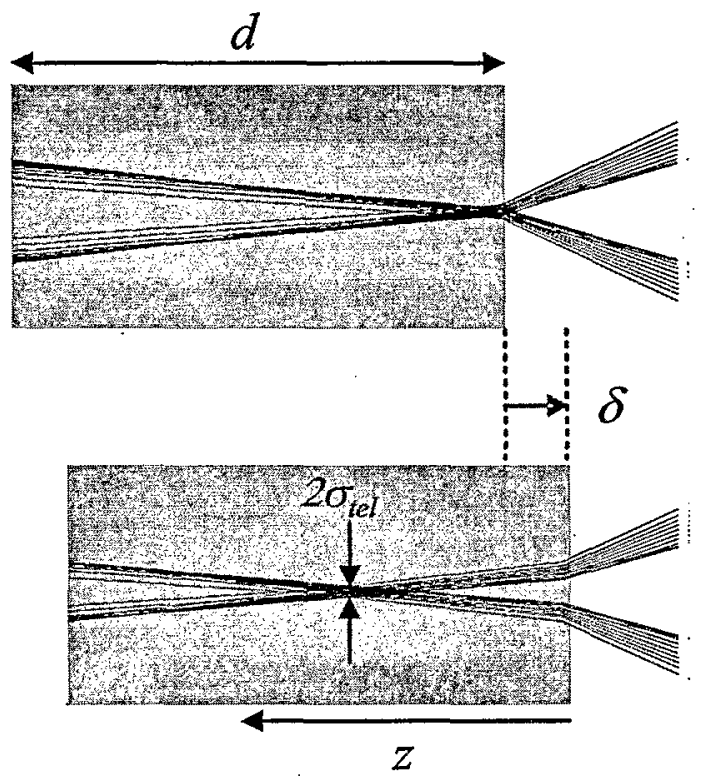

Figure 7. Geometrical model of the divergence PSF. Light is incident from the left and silicon region is shown shaded. Top: nominal focus position. Bottom: sensor displaced by $\delta$ towards the incident light.

\subsection{Combined effect of diffusion and beam divergence}

The models of section 3.1 and 3.2 can be combined to give an estimate of the PSF for various sensor configurations and bias conditions. Figure 8 shows how the PSF behaves for a 100 $\mu \mathrm{m}$-thick sensor as the focal plane is is displaced. Effects of diffusion and divergence are included. At low electric field, diffusion dominates and the short-wavelength PSF does not meet the LSST requirement. Raising the electric field to $5 \mathrm{kV} / \mathrm{cm}$ brings down the diffusion and the minimum PSF exceeds the.LSST target for all wavelengths. The focal plane has to be displaced about $15 \mu \mathrm{m}$ to produce the minimum PSF in the Y-band $(970-1060 \mathrm{~nm})$. 


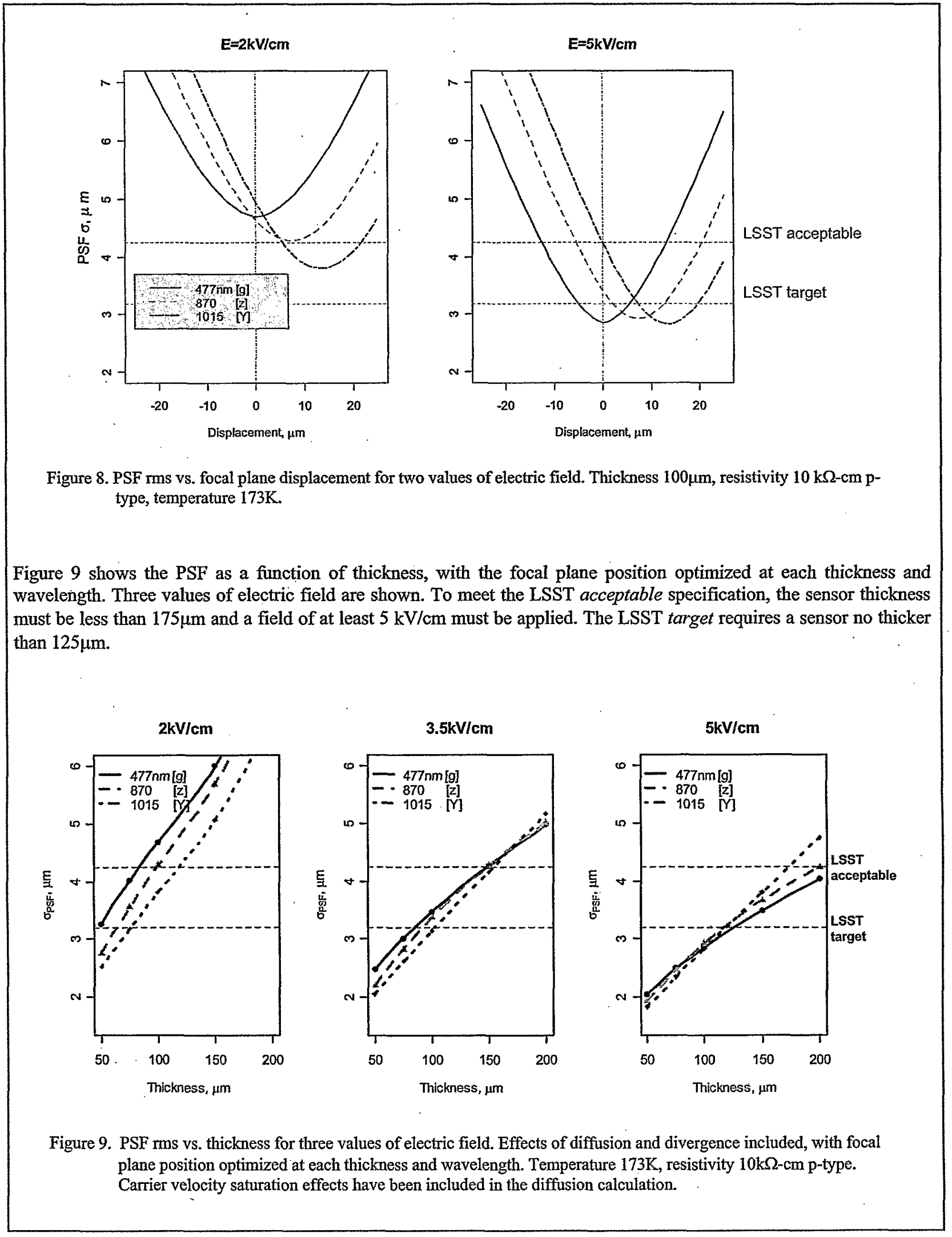

6276-75 V. 4 (p.10 of 14) / Color: No / Format: Letter / Date: 5/9/2006 2:51:52 PM 


\section{DETECTION LIMIT}

The signal-to-noise ratio for point source detectability can be written as

$$
S N R=\frac{Q R_{*} t}{\sqrt{Q R_{*} t+n_{p i x}\left[Q R_{s k y} t+R_{d a r k} t+R N^{2}\right]}}
$$

where $Q$ is the quantum efficiency, $R_{*}$ and $R_{s k y}$ are the source and sky flux at the detector respectively, $n_{p i x}$ is the effective number of pixels that contain most of the source flux, $R_{\text {dark }}$ is the dark current generation rate, $R N$ is the read noise, and $t$ is the exposure time. $n_{p i x}$ can be defined as $\pi^{2} / A_{p i x}$, where $r=$ radius around the source containing most of the light and $A_{p i x}$ is the pixel area. For Gaussian PSF, the optimum SNR occurs at $r=1.585 \sigma$, where $\sigma$ is the rms width of the PSF. In the faint source limit the signal-to-noise is thus proportional to $\frac{\sqrt{Q t / R_{s k y}}}{\sigma_{P S F}}$. We have seen that in general $Q$ and $\sigma_{P S F}$ both increase with sensor thickness, especially at near-IR wavelengths.

The point source figure of merit (8) (or equivalently the limiting magnitude for which the $\mathrm{S} / \mathrm{N}$ exceeds a desired threshold) can be calculated after making some assumptions about the LSST site, optics, and filters. Table 2 shows the telescope and sensor parameters and Table 3 gives a set of five possible filter choices for LSST [5].

Table 2. LSST telescope and sensor parameters.

\begin{tabular}{l|l}
\hline Primary mirror diameter & $8.4 \mathrm{~m}$ \\
Secondary obscuration & $61 \%$ \\
Mirror transmission per surface & $97 \%$ \\
Filter transmission per surface & $97 \%$ \\
Exposure time & $15 \mathrm{~s}$ \\
Sensor dark rate & $4 \mathrm{e}^{-/ \mathrm{pixel} / \mathrm{s}}$ \\
Sensor read noise & $10 \mathrm{e}^{-/ \mathrm{read}}$ \\
\hline
\end{tabular}

Table 3. LSST filter set, and sky brightness in each band $[5,6]$

\begin{tabular}{l|lll}
\hline Filter & lower cutoff & upper cutoff & Sky brightness \\
\hline$g$ & 402 & 552 & 22.3 \\
$r$ & 552 & 691 & 21.2 \\
$i$ & 691 & 818 & 20.0 \\
$z$ & 818 & 922 & 19.1 \\
$Y$ & 970 & 1060 & 18.4 \\
\hline & $\mathrm{nm}$ & $\mathrm{nm}$ & $\mathrm{mag} / \mathrm{arcsec}^{2}$ \\
\hline
\end{tabular}

For each sensor thickness, the expressions for QE and PSF developed in Sections 2 and 3 were averaged over the filter bandwidths and the limiting magnitude for detectability at the level of $\mathrm{SNR}=10$ was calculated from Eq. (8). For red wavelengths, we used the PSF at best focus as discussed in Section 3.3. The results are shown in Fig. 10 and Fig. 11. 

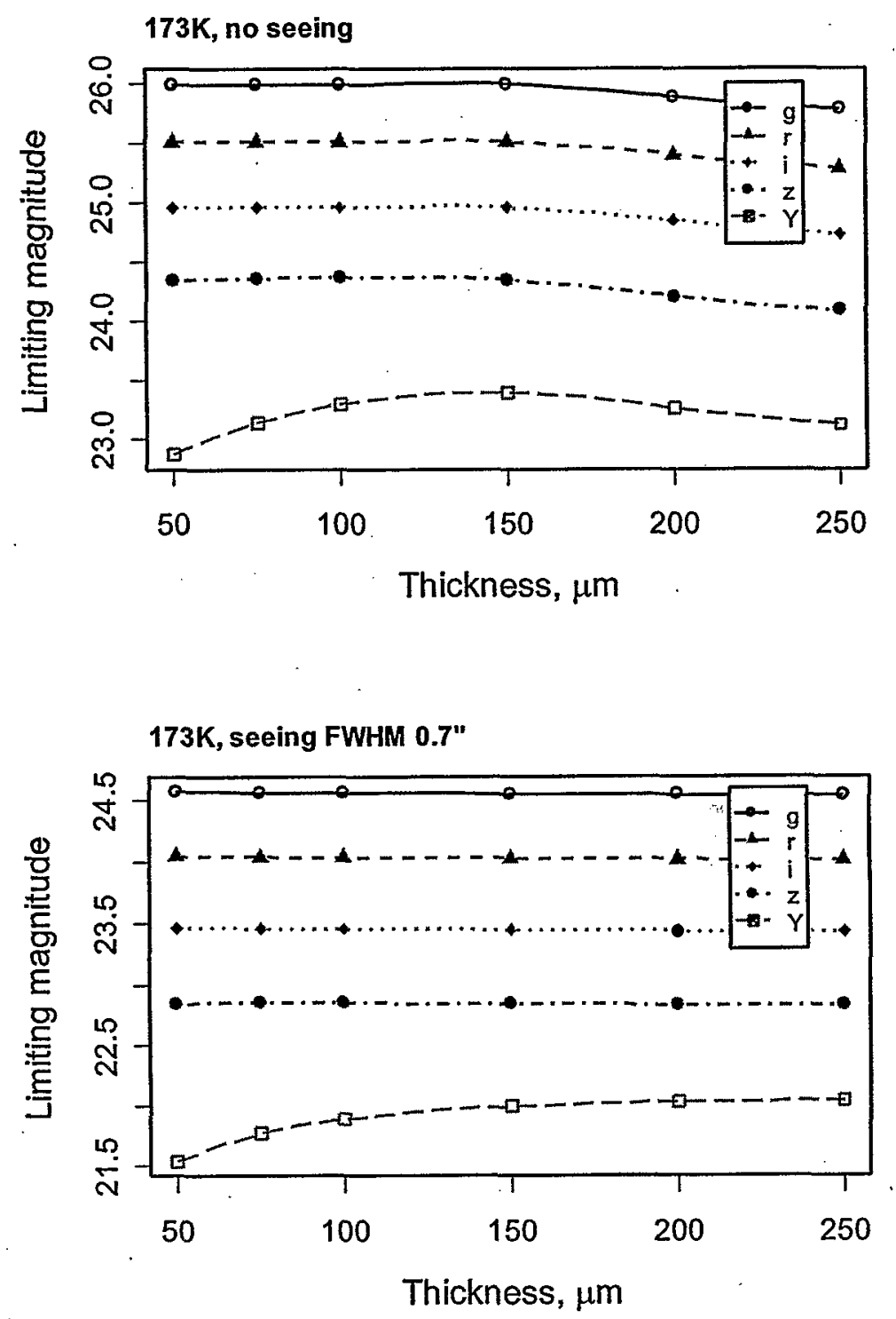

Figure 10. Limiting magnitude $(S / N=10)$ for 15-second LSST exposures, for the filter set shown in Table 3. Sensor operating temperature $173 \mathrm{~K}$; substrate $10 \mathrm{k} \Omega$-cm p-type; average electric field $5 \mathrm{kV} / \mathrm{cm}$; focal plane position adjusted for best focus in each band. Reflection losses at sensor and atmospheric extinction not included. Top: Atmospheric seeing not included; Bottom: including seeing of 0.7" FWHM. 

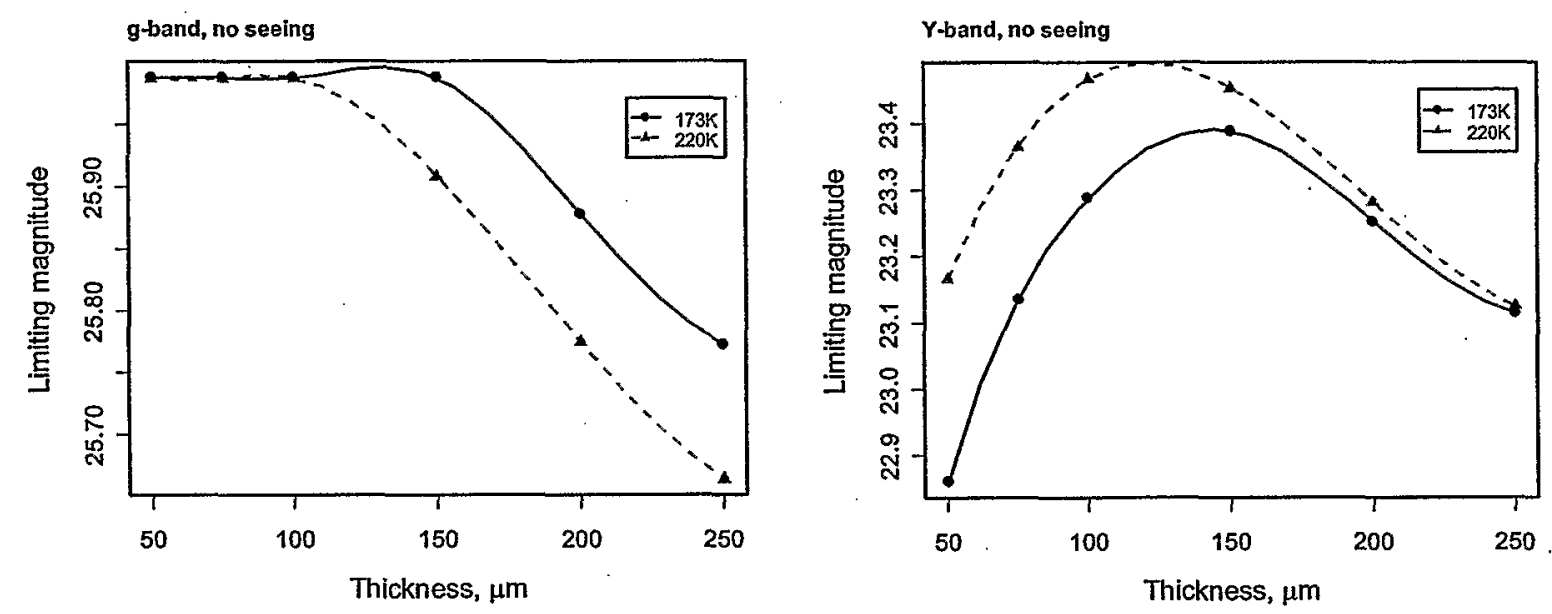

Figure 11. Limiting magnitude vs. thickness compared at two temperatures. Left: $g$-band. Right: Y-band. Same detector parameters as Fig. 10.

\section{CONCLUSIONS}

We have studied the dependence of LSST sensors on silicon thickness. Since thicker sensors have better red quantum efficiency but poorer point spread function, a compromise thickness must be chosen. The quantum efficiency has been calculated based on the known absorption length of silicon as a function of wavelength and temperature. PSF was estimated from knowledge of the high-field transport properties of carriers, and by Monte-Carlo modeling of light conversion in the sensor. The important conclusions are:

- At least $100 \mu \mathrm{m}$ thickness is needed to reach the LSST requirement for $25 \% \mathrm{QE}$ at $\lambda=1000 \mathrm{~nm}$.

- The sensor must have high resistivity and be biased well above full depletion to control the diffusion component of PSF.

- The fast focal ratio of LSST leads to an additional contribution to PSF at long wavelengths, due to the projection of the divergent cone of weakly-absorbed light.

- Because of beam divergence, there is a focus shift at long wavelengths where optimum focus is obtained with the sensor displaced in the direction of the incoming light.

- A sensor thickness of around $125-150 \mu \mathrm{m}$ gives the optimum $10 \sigma$ detection limit in Y-band.

\section{REFERENCES}

1. K. Rajkanan et al., Sol. St. Electr. 22, 793-795 (1979).

2. R. Allsman et al., "The Large Synoptic Survey Telescope (LSST), A proposal for research and development and preconstruction engineering design", submitted to Dept. of Energy Jan. 20, 2006, Table 4.3.2-2 and Table 4.3.2-3. 
3. D.E. Groom, "The red problem", LBL Internal Note, 31 October 1996. Available: http://design.lbl.gov/ccd/papers/red problem.ps.

4. D.E. Groom, "Update of depth of focus considerations for red light incident on a thick CCD in a fast optical system", LBL Internal Note, 20 March 2002. Available: http://www-ccd.lbl.gov/depth focus.ps.

5. R. Allsman, op. cit., Table 4.3.7-2.

6. A. Walker et al. "Night Sky Brightness at Cerro Pachon", 15 June 2004, NOAO newsletter \#10. Available: http://www.ctio.noao.edu/site/pachon_sky/.

\section{DISCLAMMER}

This report was prepared as an account of work sponsored by an agency of the United States Government. Neither the United States Government nor any agency thereof, nor any of their employees, nor any of their contractors, subcontractors, or their employees, makes any warranty, express or implied, or assumes any legal liability or responsibility for the accuracy, completeness, or any third party's use or the results of such use of any information, apparatus, product, or process disclosed, or represents that its use would not infringe privately owned rights. Reference herein to any specific commercial product, process, or service by trade name, trademark, manufacturer, or otherwise, does not necessarily constitute or imply its endorsement, recommendation; or favoring by the United States Government or any agency thereof or its contractors or subcontractors. The views and opinions of authors expressed herein do not necessarily state or reflect those of the United States Government or any agency thereof. The publisher by accepting the manuscript for publication acknowledges that the United States Government retains a non-exclusive, paid-up, irrevocable, world-wide license to publish or reproduce the published form of this manuscript, or allow others to do so, for United States Government purposes. This preprint is intended for publication in a journal or proceedings. Since changes may be made before publication, it may not be cited or reproduced without the author's permission. 